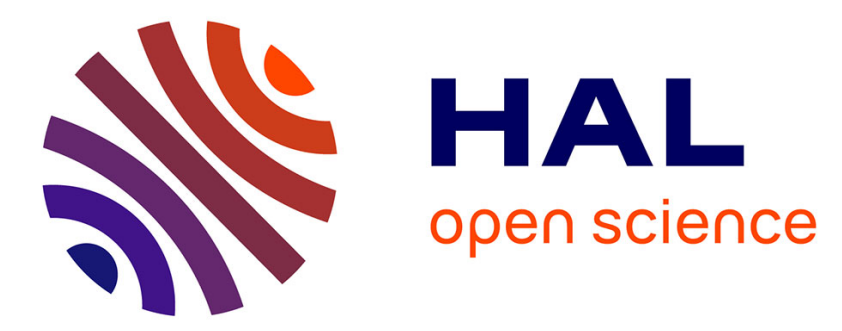

\title{
Embryons et tortues: les dépôts de fondation découverts au perron nord de la terrassse des Éléphants
}

Christophe Pottier

\section{To cite this version:}

Christophe Pottier. Embryons et tortues: les dépôts de fondation découverts au perron nord de la terrassse des Éléphants. Bulletin de l'Ecole française d'Extrême-Orient, 1997, 84 (1), pp.402-407. 10.3406/befeo.1997.3822 . halshs-01989133

\section{HAL Id: halshs-01989133 \\ https://shs.hal.science/halshs-01989133}

Submitted on 22 Jan 2019

HAL is a multi-disciplinary open access archive for the deposit and dissemination of scientific research documents, whether they are published or not. The documents may come from teaching and research institutions in France or abroad, or from public or private research centers.
L'archive ouverte pluridisciplinaire HAL, est destinée au dépôt et à la diffusion de documents scientifiques de niveau recherche, publiés ou non, émanant des établissements d'enseignement et de recherche français ou étrangers, des laboratoires publics ou privés.

\section{()ㅜ) $\Theta$}

Distributed under a Creative Commons Attribution - NoDerivatives| 4.0 International 
Embryons et tortues : les dépôts de fondation découverts au perron nord de la terrassse des Éléphants

Christophe Pottier

\section{Citer ce document / Cite this document :}

Pottier Christophe. Embryons et tortues : les dépôts de fondation découverts au perron nord de la terrassse des Éléphants . In: Bulletin de l'Ecole française d'Extrême-Orient. Tome 84, 1997. pp. 402-407;

doi : https://doi.org/10.3406/befeo.1997.3822

https://www.persee.fr/doc/befeo_0336-1519_1997_num_84_1_3822

Fichier pdf généré le 17/01/2019 


\section{Embryons et tortues : des dépôts de fondation découverts au perron nord de la terrasse des Éléphants}

On a souvent reproché aux bâtisseurs d'Angkor d'avoir négligé la structure au profit de la sculpture: artistes inspirés, architectes de génie, mais bien piètres ingénieurs. Outre que cette vision réductrice est révélatrice des limites d'une analyse occidentale empreinte d'une culture constructive étrangère au Cambodge ancien, c'est aussi faire sans doute bien peu de cas des rituels religieux qui présidaient à toute fondation, sacralisant l'œuvre et garantissant stabilité et pérennité à l'ouvrage. C'est l'importance du rituel qui nous est rappelée à l'occasion de la découverte récente d'un remarquable réseau de dépôts de fondation au perron nord de la terrasse des Éléphants.

\section{Les dépôts de fondation}

Il n'est certes pas exceptionnel de rencontrer des dépôts de fondation en place dès lors que l'on démonte une structure angkorienne. Les pilleurs qui ont sévi et sévissent encore dans les environs du site d'Angkor - et ailleurs, comme à Bantay Chmar où une dizaine ont péri en 1992 sous l'effondrement de la tour qu'ils minaient - pourraient malheureusement mais fort aisément le confirmer. Pour qui est plus scrupuleux envers le patrimoine khmer, les occasions d'étudier des dépôts in situ sont nettement plus rares, car les anastyloses intégrales ou les fouilles ne sont, après tout, pas si nombreuses. Elles n'offrent d'ailleurs pas toujours ce type de découverte, soit que le site ait déjà été pillé, soit que les dépôts utilisés lors du rituel de fondation aient été retirés avant la construction. Plus de soixante-dix « dépôts sacrés » ou « dépôts de fondation» ont été mentionnés par les divers conservateurs d'Angkor depuis le début du siècle, mais seuls les deux-tiers ont été trouvés en place (cf. tableau p. 400-401). Ces dépôts étaient placés parfois dans les superstructures (moins d'un quart des cas), mais surtout sous les piédestaux au centre des sanctuaires. Ils étaient principalement constitués de quelques petites feuilles de métaux précieux et de divers gemmes, logés dans des alvéoles réservées à cet effet dans des blocs de pierre ou des briques.

Soulignons enfin que les dépôts retrouvés in situ l'ont été dans un contexte de sanctuaire, sauf rares exceptions ${ }^{88}$. Nul dépôt n'avait été encore dégagé aux terrasses royales. Aussi, à l'occasion des travaux d'anastylose de l'extension orientale du perron nord de la terrasse des Éléphants, nous n'envisagions qu'avec circonspection d'avoir à y mettre au jour un éventuel dépôt de fondation, d'autant que l'édifice avait été en grande partie déjà démonté en 1953. Le démontage et la fouille de la fosse de fondation ${ }^{89}$ nous ont pourtant permis de mettre au jour un

88. On doit sans doute relativiser cette constatation en rappelant que les travaux et les anastyloses de la Conservation d'Angkor ne sont que rarement sortis d'un contexte de sanctuaire. Notons le statut particulier des dépôts trouvés dans la chaussée de portes d'Angkor Thom, le dépôt du Prasat Crun Sud Est et le cas à ce jour unique d'un dépôt mis au jour dans le tablier d'un pont (Rapport de la Conservation d'Angkor, décembre 1923 et BEFEO XXIII, 1923, p. 541). Que ce dernier exemple soit unique ne saurait trop étonner vu la délicatesse avec laquelle les Travaux Publics avaient détruit les nombreux ponts situés sur la route de Siemreap à Kompong Thom dans les années vingt.

89. L'anastylose conduite par H. Marchal en 1953 n'ayant pas touché aux assises basses, nous avons pu y retrouver les structures d'origine. Le système de fondation était composé d'une semelle périmétrale (deux assises de « réglage » en blocs de grès ou de latérite, grossièrement assemblés et à peine taillés) qui supportait le mur de parement extérieur et, accolé à l'arrière, un second de latérite qui 
réseau composé de onze dépôts sur une surface réduite à quelques $100 \mathrm{~m}^{2}$, tous situés près de la surface de la fosse de fondation : deux pots en bronze, trois «embryons » et six tortues de plomb (cf. fig. 2, p. 399) ${ }^{90}$.

\section{Les pots en bronze}

Les pots en bronze (P1 et P2 sur fig. 2, p.399, photo 4), bien conservés, sont de formes presque similaires (diamètre $80 \mathrm{~mm}$, hauteur $65 \mathrm{~mm}$ environ). Ils étaient placés droits, le col vers le haut, directement dans le sable de la partie supérieure de la fosse. Ils semblent avoir été vides à l'origine. L'un était situé sous l'escalier sud, l'autre dans les environs de l'escalier symétrique au nord, mais à l'extérieur de l'emprise de l'édifice.

\section{Les « embryons»}

Trois dépôts ont été mis au jour (E1 à E3 sur fig. 2, p. 399), chacun à une dizaine de centimètres sous un bloc de grès en réemploi isolé dans le remblai à la surface de la fosse de fondation. Leur localisation ne semble pourtant pas montrer de logique particulière par rapport au plan de l'édifice. Chaque dépôt se présente comme un conglomérat de plan circulaire au profil ovoïde avec fond plat (diamètre $68 \mathrm{~mm}$, hauteur $44 \mathrm{~mm}$ environ), recouvert d'une fine feuille d'un alliage métallique à base de cuivre formant une enveloppe hermétique. De nature très fragile, seul le premier conglomérat dégagé a été en partie nettoyé (E2, photos 1,2 et 3 ), ouvert et vidé de son contenu que nous décrivons ici ${ }^{91}$. La surface de l'enveloppe métallique est ornée, au moins en partie, de légers reliefs de pétales de lotus stylisés. Mais l'intérêt majeur réside dans la diversité de ce que contenait cette enveloppe: une feuille d'or $(9 \times 15 \times 0,5 \mathrm{~mm})$, une petite plaque de bronze $(14 \times 20 \times 1 \mathrm{~mm})$, deux fils d'or (longueur totale : $32 \mathrm{~mm}$ ), un cristal rose poli (améthyste ou rubellite ?), six cristaux de quartz, un petit fragment brut d'une pierre bleue, un petit nodule d'une pâte jaunâtre (résine ?), un second d'une pâte oxydéc, deux bouts de fil de soie ${ }^{92}$ (longueur d'un fil $23 \mathrm{~mm}$ ), une bobine de fil de bronze (longueur

enveloppait le remblai interne de la terrasse, constitué de sable, de terre et de gravats de taille. Tant les assises de réglage que le remblai interne reposaient sur une fosse comblée d'un sable fin assez pur.

90. Pour indication, nous donnons ci-après les cordonnées exactes des dépôts ( $\mathrm{X}$ et $\mathrm{Y}$ font référence à la trame approximativement est ouest établie pour la restauration, $\mathrm{Z}$ est rattaché au nivellement IGN 1994): P1/-18,36/-50,15/ 22,9; P2/ -21,00/-58,80/ 23,21; E1/ -23,28/-49,18/ 22,68; $\mathrm{E} 2 /-21,53 /-59,51 / 23,07, \mathrm{E} 3 /-23,10 /-59,82 / 22,70 ; \mathrm{t} 1 /-20,54 /-54,24 / 23,03 ; \mathrm{t} 2 /-20,37 /-54,86 /$ 23,01; T1/ -20,64/ -49,45/22,37; T2/ -20,24/ -53,11/ 22,58; T3/ -19,40/ -57,25/ 22,69; T4/ -20,41/ $-60,12 / 22,50$

91. Les deux derniers, bien que leurs coques soient fissurées, ont été dégagés sans être ouverts, en bloc avec le sable environnant afin de conserver au mieux les matériaux fragiles qu'ils renferment sans doute puisqu'ils semblent identiques au premier. Les trois objets de ce type ont été empaquetés et conservés dans des boîtes hermétiques.

92. Nous remercions messieurs M. Monnot (Sérica SA, ingénieur filature) et A. Peyré (Consultant, Ingénieur sériciculture) qui ont gracieusement accepté de se livrer à l'examen d'un échantillon avec les moyens limités disponibles à Siemreap. S'agissant, pour l'instant et à notre connaissance, de l'exemplaire de soie le plus ancien mis au jour au Cambodge, il nous semble adéquat de citer dans les lignes qui suivent les extraits principaux de leur rapport d'examen:

«Examen à l'œil nu: le fragment se présente comme un fil, c'est-à-dire un ensemble de fibres assemblées, en faisceau cylindrique (longueur $4 \mathrm{~mm}$, largeur environ $0,2 \mathrm{~mm}$ ). Il est légèrement torsadé (...). La couleur, terreuse, est lumineuse et homogène. Le fragment, globalement linéaire, ne présente pas de traces de tissage (non déformé par l'insertion dans une chaîne).

Examen au microscope optique (Olympus oculaire x10, objectifs $\times 10, \times 40$ et $\times 100$ ).

Au grossissement $\times 10$. Le fil apparaît homogène et dense, les fibres se touchant sans interstices. Il comporte une quarantaine de fibres individuelles juxtaposées sans liant apparent. En effet, la surface 
supérieure à $540 \mathrm{~mm}$ ), le tout aggloméré à de nombreuses graines (sésame ?) et à ce qui nous a semblé être des feuilles végétales. On doit à l'étanchéité de l'enveloppe métallique d'avoir permis la conservation exceptionnelle des matières végétales.

Une recherche sommaire d'éventuelles références dans quelques traités de construction indiens ne nous a pas permis de trouver de description concordante bien que dans le Mayamata $^{93}$, quelques passages relatifs aux rituels et aux dépôts de fondation évoquent des vases remplis d'eau parfumée (les pots en bronze retrouvés vides ?), un lacet pour un dépôt sivaïte (la bobine de bronze ?), ou un cordon sacrificiel (les fils de soie ?). Par ailleurs, Le temple selon Marîci fait mention de graines de sésame et décrit des embryons [qui contiennent] deux types d'objets : d'une part, des éléments susceptibles de croissance; d'autre part, des symboles confectionnés $d^{\prime}{ }^{\prime 94}$. Cette distinction entre végétaux et minéraux/métaux apparaît valable ici bien que les objets soient - en nombre - essentiellement du second type. Il nous a donc semblé possible en l'état actuel de considérer ces conglomérats comme des « embryons ».

\section{Les tortues de plomb}

Six tortues en plomb ont été dégagées dans le remblai de la fosse de fondation. Deux petites tortues ( $\mathrm{t} 1$ et $\mathrm{t} 2$ sur fig. 2, p. $399,161 \times 122 \times 60 \mathrm{~mm}$ et $175 \times 130 \times 70 \mathrm{~mm}$ ), sont de forme très schématique d'où seule la tête ressort. La première est assez altérée (une partie importante de la carapace manque, photo 5) et la seconde, entière, présente au sommet de sa carapace une ouverture circulaire fermée par un petit disque de plomb. Quatre grandes tortues de

du fragment est régulière, et le motif formé par la juxtaposition des fibres n'est jamais distendu ou sur épaissi par un média de liaison. De plus, la structure des fibres reste très régulière dans toute la masse du fil. Ces fibres sont parallèles entre elles, sans croisure ni enchevêtrement; seul un effet de torsion affecte l'ensemble du fragment. Les fibres apparaissent très régulières en aspect, dimension et couleur. La dimension de la fibre unitaire est estimée à environ $13 \mu$, ceci devant être confirmé par examen à l'aide d'un oculaire micrométrique (...).

$\mathrm{Au}$ grossissement $\times 100$, objectif à immersion. Les fibres restent homogènes en aspect et dimension (...). La variation de mise au point par vis micrométrique fait apparaître (...), sur les fibres isolées du pourtour et des extrémités, un nouvel aspect: une zone plus sombre, au centre de la fibre se déplace sur celle-ci avec un mouvement apparent de vrille, en restant médiane par rapport à la largeur. La fibre cylindrique paraît donc être formée de deux sous unités contigues, intimement liées, que seul un sillon central délimite. L'ensemble présente une faible torsion en vrille.

Interprétation. La description précédante correspond à celle d'une bave (fil unitaire) de ver à soie. Le bombyx du mûrier sécrète la soie à travers deux orifices (ou filières) lorsqu'il fabrique son cocon dans lequel il subira la mue imaginale. La bave de soie ainsi filée en continu se compose de deux sous ensembles de section ovaloïde, intimement liés et enrobé par une matrice. Ce filament est fin $(10 \mu)$ et trop fragile pour être utilisé seul. Les filés de soie, ou fils de soie grège, sont donc obtenus en dévidant plusieurs cocons ensembles. De plus ces fils de soie grège ont un aspect terne et un toucher rugueux. Le décreusage permet d'éliminer une grande partie de la matrice externe et de faire apparaître les torons de fibroine (protéine de la soie).

Dans le cas du fragment observé, on peut émettre l'hypothèse qu'il s'agit d'un fil de soie, suffisamment décreusé pour laisser apparaître un sillon médian. Ce fil de soie aurait été obtenu par dévidage conjoint d'une quarantaine de cocons.

Conclusion. Les examens optiques et microscopiques du fragment incitent à penser qu'il s'agit d'un fil de soie en raison: de l'aspect lisse et régulier des fibres unitaires, des aspects de surface (lisse) et d'éclat (brillant), de la juxtaposition homogène et sans accidents des fibres [et enfin] de la présence à fort grossissement d'un relief sous tendant une structure bipartite. À ce niveau d'investigations, il est impossible de conclure avec certitude; toutefois les éléments concordants (ci-dessus) permettent de retenir une nette présomption et d'orienter les recherches ultérieures vers une confirmation de cette conclusion. »

93. B. Dagens, Mayamata, IFP, Pondichéry, 1970.

94. G. Colas, Le temple selon Marîci, IFP, Pondichéry, 1986, p. 151, note 1. 
plomb (T1 à T4 sur fig. 2, p. 399, entre 45 et $65 \mathrm{~cm}$ de long, de forme assez réaliste avec la tête et les pattes en saillie, photo 6) ont été localisées grâce à la collaboration des démineurs de la COFRAS-CIDEV. Elles étaient installées au fond d'une excavation d'environ $50 \mathrm{~cm}$ de profondeur creusées à partir de la surface de la fosse de fondation, et chapeautées par un bloc de latérite ou de grès. D'aspect assez frustre mais bien conservées, elles présentent une tête, quatre pattes très schématiques et un bouchon circulaire au sommet de leur carapace. Préférant préserver l'intérieur (et l'extérieur), nous n'avons pas descellé ces disques et ignorons donc le contenu des cinq tortues complètes. Les six tortues étaient alignées sur une droite nord-sud perpendiculaire à l'axe du monument, en arrière de l'assise de réglage. Les deux petites tortues étaient situées près du croisement de cette droite avec l'axe oriental de l'édifice. Elles avaient la tête orientée au sud. Une grande tortue était orientée à l'est-nord-est (T3), les trois autres au nord-est.

Ces tortues de plomb évoquent celles en grès retrouvées dans d'autres sites angkoriens ${ }^{95}$. Outre quelques différences minimes quant à la forme des cavités sommitales ou au matériau utilisé, les tortues de plomb de la terrasse des Éléphants sont évidemment à rattacher à ces objets étroitement associés au rituel de fondation ${ }^{96}$.

À Angkor, la tortue se retrouve bien sûr comme avatar de Vishnu, en particulier à l'occasion du barattage de la mer de lait. Mais les tortues de fondation retrouvées semblent plutôt s'expliquer par le symbolisme de cet animal qui dépasse le contexte proprement khmer ${ }^{97}$ : L. Malleret indiquait «que cet animal [a] tenu un rôle important, dans les conceptions anciennes de l'Asie du Sud-Est, et peut-être faut-il rattacher les traditions qui le concernent en Chine et dans l'Inde, à la persistance ou à la migration d'antiques croyances ayant pour berceau les pays de l'Indochine et de l'Indonésie ${ }^{98} »$. De par sa forme particulière entre le carré inscrit par les quatre pattes et le cercle de la carapace, entre son plastron plat et sa carapace convexe, la tortue, à mi-chemin entre l'eau et la terre, fournit un élément transitoire pour une géométrisation de l'espace et un intermédiaire entre une assise stable et un sommet en pivot. Aussi, le thème de la tortue « répond dans l'Inde et en Chine à une signification identique dans son rôle de support, d'agent de stabilité des monts et des eaux et, par extension, de longévité ». Cette symbolique est d'ailleurs encore vivante au Cambodge.

Enfin, nous remarquerons les orientations particulières des tortues en plomb. Nous ignorons si le sud a ici une signification particulière. Mais nous remarquerons que le nord-est est une orientation singulière bien souvent marquée, par exemple dans la disposition de certains édifices à caractère funéraire. À ce sujet, G. Cœdès note à la suite de P. Mus que le «nord-est, point terminal de la pradaksinä dans la conception indienne de l'espace, permet de supposer (que le marquage du cette orientation correspond) à une évasion hors de notre univers vers le ciel ${ }^{99} »$. Les nombreuses spéculations qui pourraient découler de cette signification dépassent notre présente étude, d'autant que nous ignorons encore le contenu de ces tortues.

95. L'inventaire du dépôt de la Conservation d'Angkor récemment réalisé sous la direction de B. Dagens comporte, outre trois tortues de grès mentionnées dans le tableau p. 400-401 (Porte Nord d'Angkor Thom, Neak Péan et Kleang Nord, la quatrième, celle du Bayon DCA 3651 n'a pas été retrouvée), sept tortues de grès (Palais Royal, Sra Srang, Chau Say Tevoda et quatre d'origine indéterminée) dont deux ont une cavité triangulaire sur la carapace. Renseignements gracieusement communiqués par N. Dalsheimer.

96. Sur l'originalité du matériau utilisé, cf. supra « Nouvelles données sur les couvertures en plomb à Angkor », p. 183-220 et plus particulièrement p. 205.

97. Des représentations de tortues ont été retrouvées attachées aux fondations de sites hors du Cambodge tant à Java (Prambanan), qu'en pays Cham (Pô Nagar de Nha-trang...) ou en Thaïlande péninsulaire (Suan Por Iad) et l'on rappellera aussi les porte-stèle au Vietnam et en Chine.

98. L. Malleret, L'archéologie du delta du Mékong, t. III, EFEO, 1962, p.21.

99. G. Cœdès, «Études cambodgiennes, XXXIII - La destination funéraire des grands monuments khmers », BEFEO XL, in Articles sur le pays khmer, réimpression de l'EFEO, t. 1, Paris, 1989, p. 308, note 5 . 


\section{Perspectives}

Les dépôts de fondation se rencontrent fréquement lors des travaux d'anastylose, mais ceux retrouvés à la Terrasse des éléphants sont particulièrement nombreux et, de plus, réalisés dans des matériaux métalliques. Le réseau de tortues de plomb et la présence exceptionnelle de graines et de fibres végétales dans les «embryons " apportent de nouveaux éléments à la connaissance des rituels de fondation en vigueur à la fin de la période angkorienne. Enfin ces embryons démontrent que des vestiges de nature très périssable et d'origine non monumentale peuvent avoir été conservés jusqu'à nos jours, même s'il est certain que ces matières ont bénéficié de conditions de conservation exceptionnelles. Cette perspective permet aussi indirectement d'espérer retrouver de nouveaux types d'indices archéologiques pour appréhender la vie « civile » angkorienne.

\section{Christophe POTTIER}

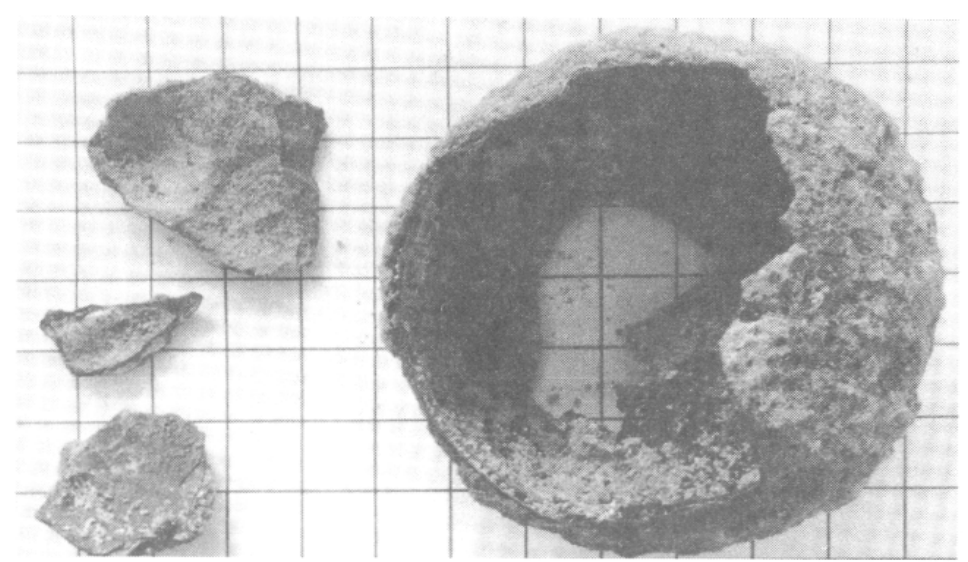

Photo 1 : dépôts de fondation sous M3 : enveloppe d'un « embryon » (E2).

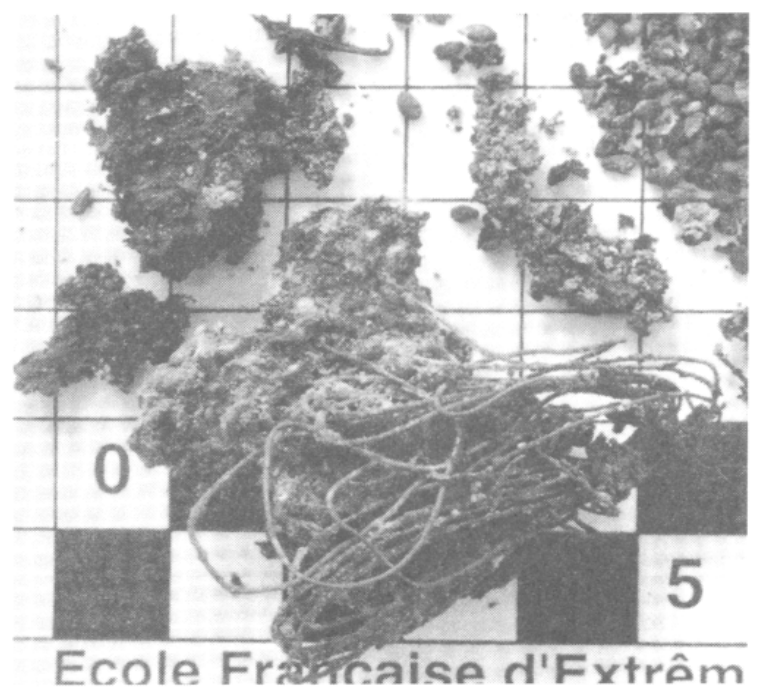

Photo 2 : dépôts de fondation sous M3 : contenu d'un « embryon », détail.

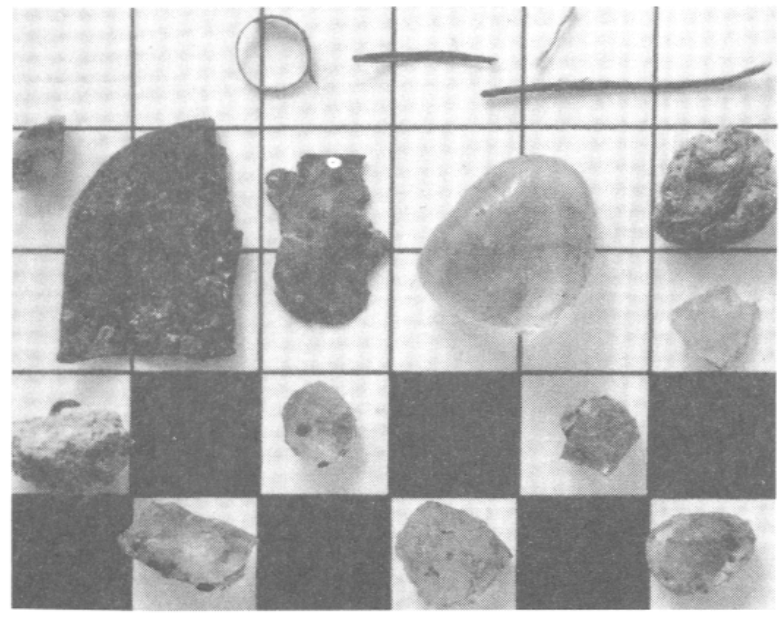

Photo 3 : dépôts de fondation sous M3 : contenu d'un « embryon », détail. 


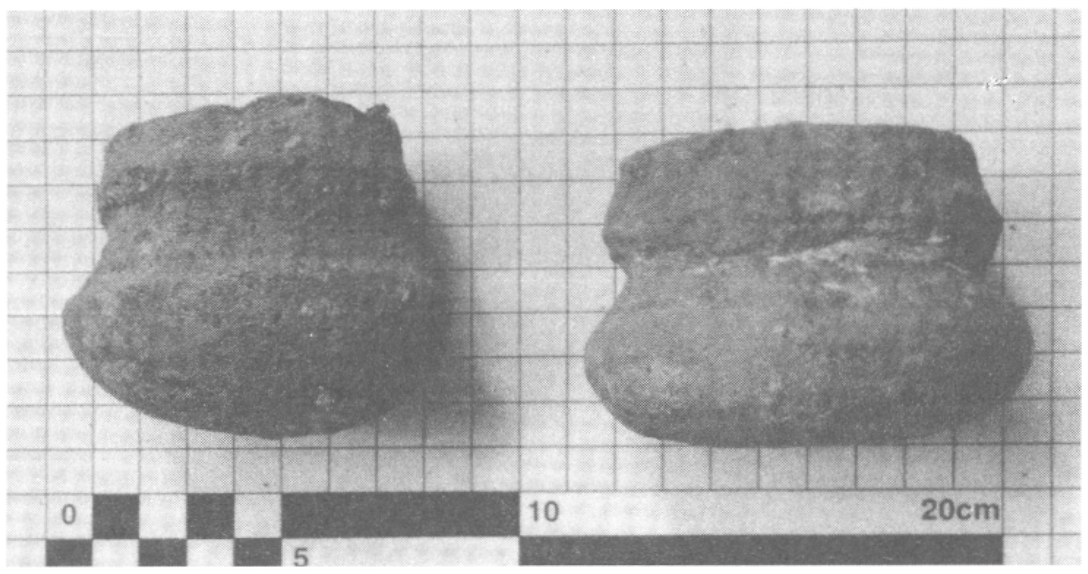

Photo 4 : dépôts de fondation sous M3: pots en bronze.

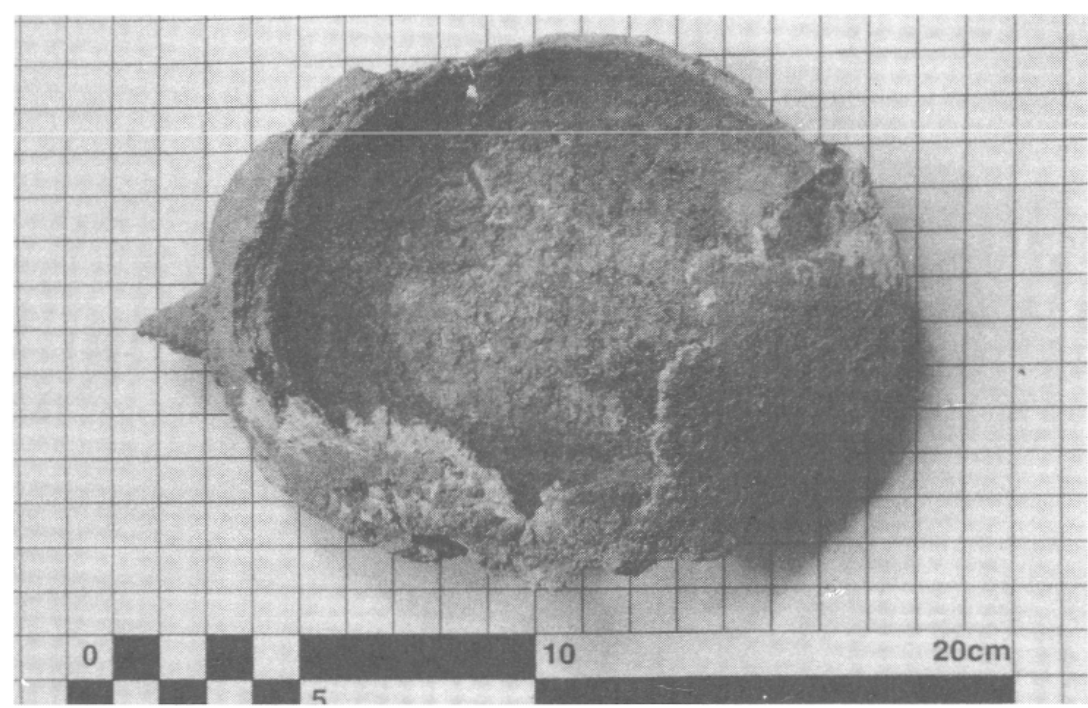

Photo 5 : dépôts de fondation sous M3: petite tortue en plomb (t1).

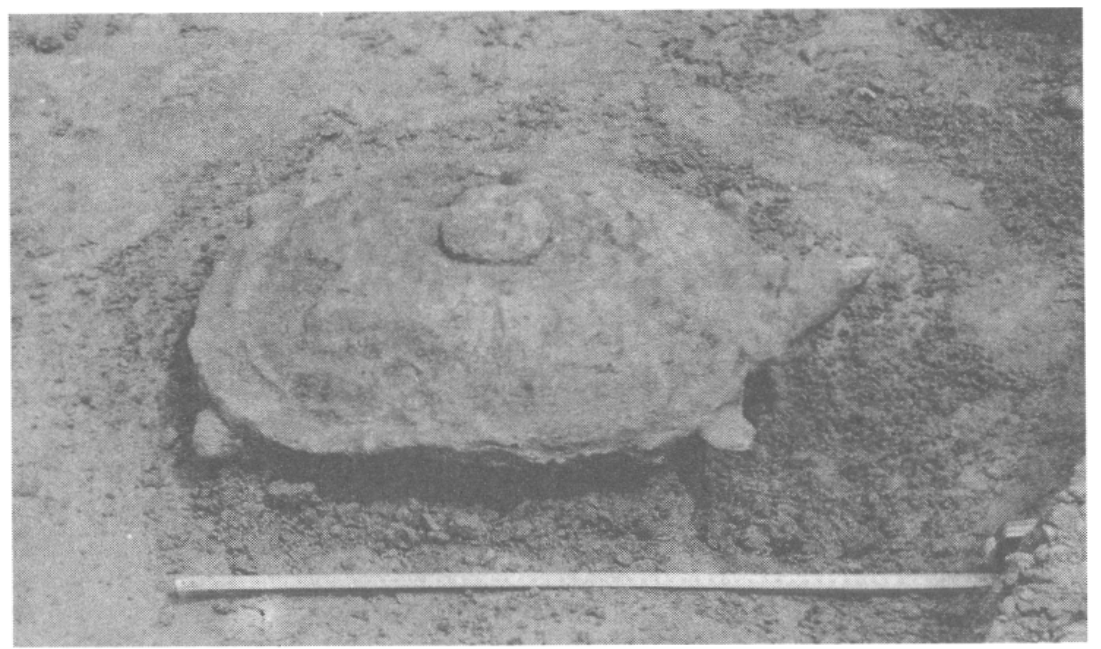

Photo 6 : dépôts de fondation sous M3: grande tortue en plomb (T2). 